http://jmscr.igmpublication.org/home/

ISSN (e)-2347-176x ISSN (p) 2455-0450

crossref DOI: https://dx.doi.org/10.18535/jmscr/v8i6.78

Journal Of Medical Science And Clinical Research

Original Article

\title{
To Study the Impact of Clinico Socio-Demographic Status for XDR Tuberculosis among MDR Tuberculosis Patients
}

Authors

\section{Dr Santosh Kumar ${ }^{1}$, Dr Gajendra Vikram Singh ${ }^{2}$, Dr Komal Lohchab ${ }^{3}$, Dr S.Hariharan ${ }^{4 *}$, Dr Amirul Haque ${ }^{5}$, Dr Vipin Kumar}

${ }^{1}$ Professor and Head, Dept of Tuberculosis \& Respiratory Medicine, S.N. Medical College, AGRA (UP)

${ }^{2}$ Associate Professor, Dept of Tuberculosis \& Respiratory Medicine SN Medical College, Agra

${ }^{3}$ Assistant Professor, Dept of Tuberculosis \& Respiratory Medicine SN Medical College, Agra

4,5,6 Junior Resident, Dept of Tuberculosis \& Respiratory Medicine SN Medical College, Agra

*Corresponding Author

\section{Dr S.Hariharan}

\begin{abstract}
Background: Tuberculosis (TB) is a chronic infectious disease that has afflicted mankind for 5000 years. It has caused more deaths in adults than any other infectious disease The emergence of multidrug-resistant (MDR) TB has introduced challenging, but surmountable, complexities to TB programs, MDR-TB remains a public health crisis and a health security threat.. XDR-TB is more expensive and difficult to treat than MDR-TB and outcomes much worse with higher rates of treatment failure and death. Therefore we planned this study to analyze the various clinico socio demographic factors associated with the development of XDR-TB among MDR-TB patients

Objective

1) To assess the various clinico socio demographic status of MDR cases

2) To identify the associated factors for the conversion of MDR to XDR tuberculosis.

3) To estimate the conversion rate of XDR tuberculosis from MDR tuberculosis

Method: It was a hospital based observational and prospective study. Informed consent of the patient was taken for the study. All information to accomplish objectives was collected by personal interview of each of the study subjects for about 30 to 45 minutes at PMDT site using pre-designed and pre-tested Proforma.

Results: Among the study participants 66.5\%(125) were males and 33.5\%(63) were females. Among males $16.8 \%$ (21) got converted to XDR while among the females 33.3\%(21) converted to XDR tuberculosis. In the study $39.9 \%$ (75) were living in houses without overcrowding and 60.1\%(113) were living in houses with overcrowding. . Among those living in overcrowded houses $14.7 \%(11)$ converted to XDR. In those who didn't live in overcrowded houses $27.4 \%(31)$ converted to XDR.

Keywords: $M D R-T B, X D R-T B$, clinico socio demographic impact, associated factors, conversion rate.
\end{abstract}

\section{Introduction}

Tuberculosis (TB) is a chronic infectious disease that has afflicted mankind for 5000 years. Its etiologic agent, known as Mycobacterium tuberculosis or Koch's bacillus, has caused more deaths in adults than any other infectious disease. Chemotherapy is the most effective weapon against TB, leading to a cure in almost all cases. However, various factors may negatively affect outcomes. 
India accounts for one fourth of the global TB burden. In 2018, an estimated 27.4 lakh cases occurred and 4.1 lakh people died due to tuberculosis (TB). An estimated 1.35 lakh incident multi-drug resistant TB (MDR-TB) patients emerge annually in India. Globally India has highest burden of both TB and MDR-TB based on estimates reported in Global TB Report $2018^{(1)}$.

Multidrug-Resistant TB (MDR-TB), defined as TB caused by Mycobacterium tuberculosis resistant to at least Isoniazid and Rifampicin, which threatens communities throughout the world. Globally, about three per cent of all newly diagnosed patients have MDR-TB.

Extensively Drug Resistant TB (XDR-TB) is resistant to at least to Isoniazid and Rifampicin, as well as to at least one fluoroquinolone and at least any of the second-line injectables which include kanamycin, amikacin and capreomycin ${ }^{(2)}$.

In 2017, MDR-TB remains a public health crisis and a health security threat. WHO estimates that there were 558,000 new cases with resistance to Rifampicin - the most effective first-line drug, of which $82 \%$ had MDR-TB. The MDR-TB burden largely falls on 3 countries - India, China and the Russian Federation - which together account for nearly half of the global cases. About $8.5 \%$ of MDR-TB cases had extensively drug-resistant TB (XDR-TB) in 2017.

The sporadic appearance and subsequent selection of drug resistant M.tuberculosis mutants is an unintended and unavoidable consequence of using anti-tuberculosis (TB) antibiotics ${ }^{(1)}$. The emergence of multidrug-resistant (MDR) TB has introduced challenging, but surmountable, complexities to TB programs that have responded by treating MDRTB with second-line drugs.

XDR-TB is more expensive and difficult to treat than MDR-TB and outcomes much worse with higher rates of treatment failure and death. These patients also presented longer duration until culture conversion. The most relevant characteristics associated with treatment failure of XDR-TB were male gender, HIV infection and resistance to all second-line drugs. Others report that MDR-TB and drug resistance to first-line drugs beside rifampicin and isoniazid are predictors of worst outcomes.

Identifying baseline patient characteristics and MDR-TB treatment-related factors associated with developing XDR-TB is crucial to the design and implementation of strategies that minimize the development of XDR-TB among individuals receiving MDR-TB therapy.

Therefore we planned this study to analyze the various clinico socio demographic factors influencing development of XDR-TB among MDR-TB patients attending Nodal DRTB centre site at Department of tuberculosis and Respiratory Medicine, S.N Medical College, Agra, Uttar Pradesh, from 1st March 2018 to 31st October 2019.

\section{Objectives}

$>$ To assess the various clinico socio demographic status of MDR cases

$>$ To identify the associated factors for the conversion of MDR to XDR tuberculosis.

$>$ To estimate the conversion rate of $\mathrm{XDR}$ tuberculosis from MDR tuberculosis

\section{Material and Methods \\ Study Design}

This study was a hospital based, observational and prospective study, which was performed at the Department of TB \& respiratory diseases S. N. Medical College Agra. The microbiological work of the study were conducted at the Department of Microbiology and Molecular Biology, National JALMA Institute for Leprosy and Other Mycobacterial Diseases (NJIL\&OMD), Agra (UP), India.

Study Period: The study was carried out from March 2018 to October 2019.

Study Population: Self-reporting and /or referred CBNAAT confirmed MDR-TB patients registered under the DOTS-Plus Project at the department of TB \& chest diseases S. N. Medical College Agra were encouraged to participate in present study. 


\section{Inclusion Criteria}

$>$ Known cases or already diagnosed cases of MDR tuberculosis patients on the basis of clinical history, sputum smear examination, physical examination, and chest radiographs and confirmed by CBNAAT, Gene-Xpert, or by drug sensitivity testing for M.tuberculosis or by Line Probe Assay (LPA).

$>$ Patient volunteers to give 2 sputum samples to look for conversion rate at end of 1 year of treatment.

$>$ Persons who provided written informed consent.

\section{Exclusion Criteria}

$>$ Any MDR TB patients who were registered under Dots Cat-IV outside the period of study.

$>$ Those who didn't give consent to participate for study.

\section{Data Collection}

All information to accomplish objectives was collected by personal interview of each of the study subjects for about 30 to 45 minutes at PMDT site using pre-designed and pre- tested Proforma. A detailed personal history of Occupation, Educational and Socioeconomic status, addiction habit and religion was also assessed.

\section{Statistical Analysis}

Data thus collected was entered into Microsoft excel 2010 worksheet in the form of master chart. Then data were tabulated and analysed as per the aims and objectives with help of appropriate statistical software (Primer statistical software version 6). Microsoft Word and Excel have been used to generate graphs, tables etc. Chi square test and fischers test were applied between various variable and drug resistance pattern.

\section{Results}

In the study group majority 58\% (109) were adults in the age group of 25- 59 years, followed by
$35.6 \%$ (67) in 15- 24 years (youth) age group, $3.5 \%$ (7) in geriatric age group. (Fig 1). The mean age of the study participants was $31.27 \pm 13.48$ years. The majority $66.5 \%$ (125) of participants were males and 33.5\% (63) were females. (Fig 2)

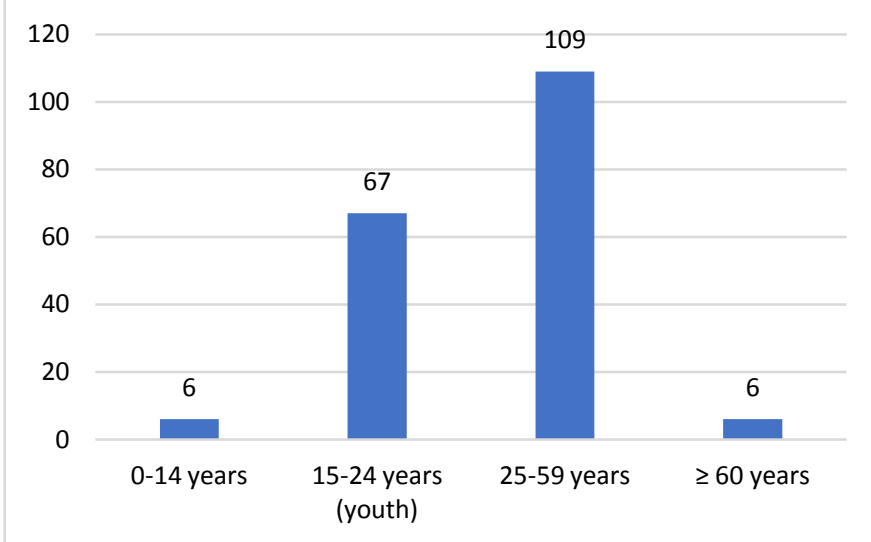

Fig 1 Distribution of participants according to age category $(\mathrm{N}=188)$

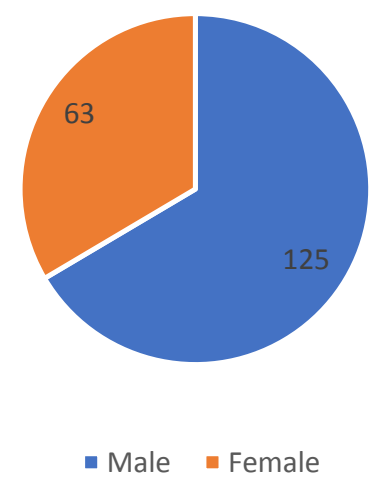

Fig 2.Gender distribution $(\mathrm{N}=188)$

There was a significant association between the gender and drug resistance of the TB bacteria $(\mathrm{p}=0.010)$. Higher proportion of females were XDR than the males. (Table 1)

Table 1: Association between the gender and the drug resistance of the TB bacteria

\begin{tabular}{|l|c|c|c|}
\hline Gender & XDR & MDR & $\mathrm{p}$ value \\
\hline Male & $21(16.8)$ & $104(83.2)$ & 0.010 \\
\cline { 1 - 3 } Female & $21(33.3)$ & $42(66.7)$ & \\
\hline
\end{tabular}

\section{Social Characteristics}

\section{Education}

The study group comprised majority $35.6 \%$ (68) people who had an education level of high school 
and above, followed by $28.7 \%$ (54) illiterates, $17.6 \%$ (33) had completed middle school and $14.9 \%$ (28) had completed primary school.

\section{Occupation}

The occupation of the participants was categorized into 5 major categories and all other occupations were grouped under others. Majority of the participants $21.2 \%$ (40) were students. There were $14.4 \%$ (27) people in labourer category (daily wage labourer, shoe maker, shoe fitter, shop keeper, painter, welder, watchman, hotel server, carpenter, bangle maker), followed by $26.6 \%$ (50) in others category, $8.5 \%$ (16) were farmers and $13.3 \%(25)$ housewife.

\section{Living Condition and Overcrowding}

$120(63.8 \%)$ people were living in urban area, followed by $61(32.4 \%)$ in rural area and $7(3.7 \%)$ in semi urban area. (Table 2). One hundred and 75 (39.9\%) were living in houses without overcrowding and 113(60.1\%) were living in houses with overcrowding. (Table 2,Fig 3)

Table 2 Living conditions \& Overcrowding status of the study participants $(\mathrm{N}=188)$

\begin{tabular}{|l|c|c|}
\hline & Frequency & Percentage \\
\hline Living conditions \\
\hline Rural & 61 & 32.4 \\
\hline Semi urban & 7 & 3.7 \\
\hline Urban & 120 & 63.8 \\
\hline Overcrowding & \\
\hline Yes & 75 & 39.9 \\
\hline No & 113 & 60.1 \\
\hline
\end{tabular}

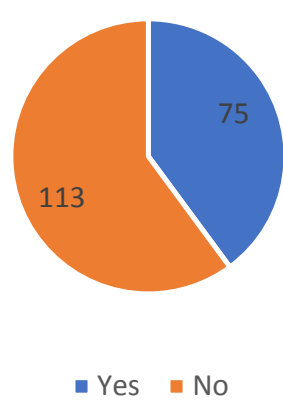

Fig 3 Distribution of participants according to presence of overcrowding in their house $(\mathrm{N}=188)$ There was a significant association between the overcrowding and drug resistance of the TB bacteria $(\mathrm{p}=0.040)$. (Table 3$)$
Table 3: Association between the overcrowding and the drug resistance of the TB bacteria

\begin{tabular}{|l|c|c|c|}
\hline Overcrowding & MDR & XDR & p value \\
\hline Yes & $64(85.3)$ & $11(14.7)$ & 0.040 \\
\cline { 1 - 3 } No & $82(72.6)$ & $31(27.4)$ & \\
\hline
\end{tabular}

Among those who have h/o cough the median duration was 6 months and IQR was 2-18 months (Table 4.1).

Among the 94 (50\%) participants with positive Xray findings, $9(4.8 \%)$ of them had unilateral findings and $85(45.2 \%)$ had bilateral X-ray findings (Table 4.2)

Table 4.1 Distribution of participants according to presence of presumptive TB symptoms and x-ray features

\begin{tabular}{|l|c|c|}
\hline $\begin{array}{l}\text { Presumptive TB } \\
\text { symptoms }\end{array}$ & Frequency & Percentage \\
\hline Cough $(\mathrm{N}=188)$ & 156 & 83 \\
\hline Present & 32 & 17 \\
\hline Absent & 137 & 72.9 \\
\hline h/o loss of weight (N=183) & 27.1 \\
\hline Present & 46 & \\
\hline Absent &
\end{tabular}

Table 4.2 Distribution of study participants according to the side of the $\mathrm{X}$ ray findings $(\mathrm{N}=94)$

\begin{tabular}{|l|c|c|}
\hline Side of X ray findings & Frequency & Percentage \\
\hline Unilateral (cavitation) & 9 & 9.5 \\
\hline Bilateral & 85 & 90.5 \\
\hline
\end{tabular}

157 (97.9\%) had HIV co infection with tuberculosis and $4(2.1 \%)$ did not have HIV co infection.

Majority $84 \%(158)$ were non-smoker and $16 \%$ (30) were smoker.

Majority $87.8 \%$ (165) were non-alcohol and $12.2 \%$ (23) were alcoholics.

Majority of our participants $77.7 \%$ (146) remained MDR, while $22.3 \%$ (42) turned into XDR during the follow-up period (Table 5).

Table 5 Distribution of participants according Drug sensitivity of XDR status $(\mathrm{N}=188)$

\begin{tabular}{|l|c|c|}
\hline XDR Status & Frequency & Percentage \\
\hline Yes & 42 & 22.3 \\
\hline No & 146 & 77.7 \\
\hline
\end{tabular}




\section{Discussion}

The present study was conducted in the Nodal DR-TB centre at the department of Tuberculosis and Chest Diseases, S.N. Medical College, Agra for a period of 18 months. A total of 188 MDR tuberculosis cases were enrolled into the study. The study participants were subjected to a detailed clinical history, thorough clinical examination, sputum examinations (including DST, LPA) and radiology.

In the present study the majority of study participants $58 \%$ were adults in the age group 25 59 years, followed by $35.6 \%(67)$ in 15-24 years (youth) age group, 3.5\%(7) in geriatric age group. The mean age was $31.27+-13.48$ years. Most of the study participants $66.5 \%(125)$ were males and $33.5 \%$ (63) were females. In our study the male : female ratio is nearly $2: 1$. This might be because of the fact in north India; even nowadays females are given less health care than males.

Among the study participants 66.5\%(125) were males and 33.5\%(63) were females. Among males $16.8 \%(21)$ got converted to XDR while among the females $33.3 \%(21)$ converted to XDR tuberculosis. In present study There was a significant association between the gender and drug resistance of the TB bacteria $(\mathrm{p}=0.010)$. Higher proportion of females were XDR than the males. (Table 1)

J.F.Gallo et $a l^{(3)}$ conducted a study in brazil where they demonstrated a higher percentage of females among XDR group ${ }^{(4,5,6)}$, this may be due to because women find great barriers for healthcare ${ }^{(4)}$ and suffer from stigma and social consequences of this disease compared to men ${ }^{(7)}$. All these factors leads to delay in diagnosis and treatment, which would facilitate the development of drug resistance. In another study done in South Korea $^{(4)}$, China ${ }^{(8)}$ and South Africa ${ }^{(9)}$ they found that the percentage of female in the XDR group were significantly higher than males. Our study findings were in accordance with these studies in regards to higher proportion of female gender in the XDR group.
In the present study $39.9 \%$ (75) were living in houses without overcrowding and $60.1 \%$ (113) were living in houses with overcrowding. (Table 3, Fig 6). Among those living in overcrowded houses $14.7 \%$ (11) converted to XDR. In those who didn't live in overcrowded houses $27.4 \%$ (31) converted to XDR. In our study we found out that there was a significant association between the overcrowding and drug resistance of the TB bacteria $(\mathrm{p}=0.040)$. (Table 3$)$.

J.F.Gallo et $a l^{(3)}$ found out that overcrowding especially in urban cities due migration as a result of industrialization leads to drug resistant transmission $^{(10)}$ in households or between neighbors. Yanina et $a l^{(11)}$ found out that urban resident are at higher risk of acquiring resistant TB due to overcrowding. These two paper were in accordance with the results of our study that there was a significant association between overcrowding and drug resistance $(p, 0.040)$.

In the current study of 188 participants all were confirmed MDR-TB patients either by CBNAAT or by LPA or by culture, among them we found out that $22.3 \%$ (42) converted to XDR ie they developed resistance to fluroquinolone and a second line injectable and the remaining participants of the study $77.7 \%$ (146) remained as MDR(Table 3).

In the study of porwal et $a l^{(10)}$ the prevalence of MDR was $3.7 \%$. Myneedu et $a l^{(32)}$ reported that $20 \%$ prevalence of XDR among MDR patients. Mondal et $a l^{(33)}$ demonstrated 7.4\% XDR among MDR strains. A study from Hinduja hospital Mumbai $^{(34)}$ revealed $11 \%$ MDR strains ad XDR. In Sameer Adwani et $a l^{(35)}$ found $4.85 \%$ of their MDR patient as having XDR. Sharma et $a l^{(36)}$ reported a $33.3 \%$ of XDR-TB in a population of HIV sero-positive MDR-TB patients from AIIMS, Delhi. Only one population based study was done by then TRC Chennai and found out the prevalence of XDR to be $1.5 \%{ }^{(57)}$. However in the study we found that there a was high prevalence of XDR-TB $22.3 \%$ among MDR-TB than the national survey suggest. 
In our study only female gender and overcrowding emerged as risk factors among various clinico socio demographic factors and the conversion rate to was $22.7 \%$.

\section{Conclusion}

To conclude we documented a significant risk of XDR-TB which was associated with female gender and overcrowding. More detailed population-based studies are required to know about the full burden of MDR/XDR-TB at a community level. Knowing the risk factors and drug resistant pattern for XDR-TB could help initiate the TB programmes to devise effective, fast detection methods and new treatment regimens and improve the outcomes of MDR tuberculosis, the need for these are crucial especially in India where drug-resistant tuberculosis is spreading like wildfire.

\section{Bibliography}

1. World Health Organization. Global tuberculosis report, 2018. WHO/HTM/ TB/2018.11. Geneva, Switzerland: WHO, 2018.

2. Extensively drug-resistant tuberculosis (XDR-TB): recommendations for prevention and control. Weekly Epidemiol Rec 2006; 81: 430-432

3. J.F. Gallo, J.M.W. Pinhata, V. Simonsen, V.M.N. Galesi, L. Ferrazoli, R.S. Oliveira, Prevalence, associated factors, outcomes and transmission of extensively drug-resistant tuberculosis among multidrug-resistant tuberculosis patients in Sao Paulo, Brazil: a crosssectional study, Clinical Microbiology and Infection 2018;24:E889e895.

4. Christie Y. Jeon, A Soo Hee Hwang, A Jin Hong Min, D. Rebecca Prevots, Lisa C. Goldfeder, Hyeyoung Lee, Seok Yong Eum, Doo Soo Jeon, Hyung Seok Kang, Jin Hee Kim, Byoung Ju Kim, Dae Yeon Kim, Steven M. Holland,Seung Kyu Park, Sang Nae Cho, Clifton E. Barry, 3rd, and
Laura E. Via. Extensively Drug-Resistant Tuberculosis in South Korea: Risk Factors and Treatment Outcomes among Patients at a Tertiary Referral Hospital. Clinical Infectious Diseases 2008;46:42-9.

5. Dalton T, Cegielski P, Akkisilip S, Asencios L, Campos Caoili J, Cho SN et al. Prevalence of and risk factors for resistance to second line drugs in people with multidrug-resistant tuberculosis in eight countries: a prospective cohort study. Lancet 2012;380:1406-17.

6. Matteeli A, Roggi A, Carvalho ACC. Extensively drug-resistant tuberculosis: epidemiology and management. Clin Epidemiol 2014;6:111-8.

7. Thorson A, Johansson E. Equality or equity in health care access: a qualitative study of doctor explanation to a longer delay among female TB patients in Vietnam. Health Policy 2004;68:37-46.

8. Shenjie Tang, Shouyong Tan, Lan Yao, Fujian Li, Li Li, Xinzhi Guo, Yidian Liu, Xiaohui Hao, Yanqiong Li, Xiuxiu Ding, Zhanjun Zhang, Li Tong, Jianan Huang. Risk Factors for Poor Treatment Outcomes in Patients with MDR-TB and XDR-TB in China: Retrospective Multi Center Investigation. PLOS ONE December 2013; 8(12):E82943.

9. O'Donnell MR, Zelnick J, Wermer L, Master I, Loveday et al. Extensively drug resistant tuberculosis in women, KwaZuluNatal, South Africa. Emerg Infect Dis 2011;17(10):1942-1945.

10. Abubakat I, Zignol M, Falzon D, Raviglione M, Ditiu L, Masham S, et al. Drug-resistant tuberculosis time for visionary political leadership, Lancet Infect Dis 2013;13:529-39.

11. Yanina Balabanova, Birute Radiulyte, Edita Davidaviciene, Richard Hooper, Olga Ignatyeva, Vladyslav Nikolayevskyy and Francis A. Drobniewsk. Risk factors for drug-resistant tuberculosis patients in 
Lithuania, 2002-2008. Euro Respair J 2010;39(5):1266-1269.

12. Chhavi porwal, Amit Kaushik, Nayani Makkar, Jayant N. Banavaliker, Mahumud Hanif, Rupak Singla, Anuj K. Bhatnagat, Digambar Behera, Jitendra Nath Pande, Urvashi B. Singh. Incidence and Risk factors for extensively drug resistant tuberculosis in delhi region. PLOS ONE 2013;8:e55299

13. Myneedu VP, Visalakshi P, Verma AK, Behera D, Bhalla $\mathrm{M}$, prevalence of XDR TB cases - a retrospective study from a tertiary care TB hospital. Indian $\mathbf{J}$ Tuberc 2011;58(2):54-9.

14. Mondal R, Jain A, Extensively drugresistant Mycobacterium tuberculosis, India. Emer Infectious Dis 2007;13(9):1429-31.

15. Ajbani K, Rodrigues C, Shenai S, Mehta A, Can mutation detection accurately predict XDR-TB: Study from a tertiary care centre India. J Clin Microbiol 2011;49(4):1588-90.

16. Sameer A, Unnati D, Desai, Jyotsna M, Joshi. Prevalance of pre-extensively drugresistant tuberculosis (pre XDR-TB) and extensively drug-resistant tuberculosis (XDR-TB) among pulmonary multi drugresistant tuberculosis (MDR-TB) at a tertiary care centre in Mumbai. Journal of Krishna institute of medical sciences university 2016;5(3):13-19.

17. Singh S, Sankar MM, Gopinath K. High rate of extensively drug-reisitant tuberculosis in Indian AIDS patients. AIIDS 2007;21(7):2345-

18. Thomas A, Ramachandran R, Rehaman F, Jaggarajamma K, Santha T, Selvakumar N et al. Management of multidrug-resistant tuberculosis in the field: Tuberculosis research centre experience. Indian $\mathrm{J}$ Tuberc 2007;54:117-24. 